\section{Response to Gammal et al.}

Gammal and colleagues envision a future of medical practice in which patients undergo preemptive panel pharmacogenetic testing early in the life course, in a health-care system with the infrastructure to support the evidence-based use of that information over the subsequent decades of their health care. ${ }^{1}$ They argue that the "pivotal" question for clinical practice will be how to use existing pharmacogenetic information in patient care, not whether to order a pharmacogenetic test. Here, the efforts by the Clinical Pharmacogenetics Implementation Consortium (CPIC) are invaluable, using expert review of the best available evidence to make drug choice and dosing guidelines for clinicians when a patient's genotype is known. ${ }^{2}$ Many of us being CPIC members ourselves, we too are excited about the possibility of this future.

However, the vast majority of patients in the United States and worldwide do not currently receive health care in such a system. Until that future is realized, the pivotal question for most clinicians is, in fact, whether to order a pharmacogenetic test. At the institutional level, health-care systems are having to develop policies now about whether and how to incorporate pharmacogenetic testing in their current care models. Our policy recommendations for the Veterans Health Administration (VHA) ${ }^{3}$ arose from the urgent need among clinicians, pathology and laboratory medicine service chiefs, and other stakeholders for guidance on how to do so. For its part, CPIC explicitly states it does not make recommendations about whether to order a pharmacogenetic test. ${ }^{2}$

The questions of whether to order a clinical test in the first place and whether to use existing information in medical decision-making share similarities but have important differences. Both rely on the assertion that the test result has high clinical validity and clinical utility. The expert work by CPIC has clearly demonstrated the clinical validity of dozens of drug-gene associations. It was for this reason that the VHA Clinical Pharmacogenetics Subcommittee found the authoritative guidelines of CPIC to be an invaluable starting point as we made our policy recommendations for the VHA context. We would then argue that the clinical utility of a pharmacogenetic test requires a demonstration that prospectively collected patient outcomes are improved with its clinical use. ${ }^{3}$ For example, we strongly recommended $H L A-B$ testing prior to treatment with abacavir based on randomized controlled trial (RCT) data demonstrating elimination of hypersensitivity reaction with genotype-guided therapy. ${ }^{4} \mathrm{At}$ the other end of the spectrum, we did not routinely recommend SLCO1B1 genotyping prior to simvastatin initiation, due in part to the absence of evidence demonstrating lower rates of statin-associated muscle symptoms after genotype-guided therapy. ${ }^{5}$ While RCTs are the gold standard for such evidence, other study designs using pre/post comparisons or historical or concurrent control groups can also provide high-quality information. ${ }^{6}$ Policymakers may impose a higher threshold for the clinical utility evidence needed to support the ordering of a pharmacogenetic test in the first place compared with the use of existing pharmacogenetic information for medical decision-making, but both instances require some demonstration of improved patient outcomes.

Beyond clinical utility, the question of whether to order a test in a given health-care system also touches on issues of laboratory capacity, information technology services, clinician support, and cost, among many others. Although our policy recommendations did not explicitly consider costs, it is important to note that the meaningful use even of existing pharmacogenetic results is not "free" for a health-care system, as it still requires significant investments in the development and maintenance of health record systems enabling such use. ${ }^{7}$

We look forward to reports from ongoing research and implementation projects that will contribute to the growing evidence base for the clinical utility and feasibility of pharmacogenetic testing. We urge these projects to disseminate high-quality evidence regarding patient outcomes as it becomes available. The specific recommendations our Subcommittee made will change with the evolving evidence, but we maintain that our prioritization of improved patient outcomes is a durable approach to policymaking for healthcare systems.

\section{DISCLOSURE}

The Indiana University Pharmacogenomics Laboratory is a fee-forservice clinical laboratory. The authors declare no other conflicts of interest.

Publisher's note: Springer Nature remains neutral with regard to jurisdictional claims in published maps and institutional affiliations.

Jason L. Vassy, MD, MPH (1) ${ }^{1,2,3}$, Annjanette Stone, BS John T. Callaghan, $M D, P h D^{5,6}$, Margaret Mendes, PharmD ${ }^{7}$, Laurence J. Meyer, $M D, P h D^{8}$, Victoria M. Pratt, $P h D^{6}$, Ronald M. Przygodzki, $M D^{9}$,

Maren T. Scheuner, MD, MPH $H^{10,11}$,

Jessica Wang-Rodriguez, $M D^{7,12}$ and

Steven A. Schichman, $\mathrm{MD}, \mathrm{PhD}^{4}$ for the VHA Clinical Pharmacogenetics Subcommittee

${ }^{1}$ Section of General Internal Medicine, VA Boston Healthcare System, Boston, MA, USA; ${ }^{2}$ Department of Medicine, Harvard Medical School, Boston, MA, USA; ${ }^{3}$ Division of General Internal Medicine and Primary Care, Brigham and Women's Hospital, Boston, MA, USA; ${ }^{4}$ Pharmacogenomics Analysis Laboratory, Central Arkansas 
Veterans Healthcare System, Little Rock, AR, USA; ${ }^{5}$ Richard L. Roudebush VA Medical Center, Indianapolis, IN, USA; ${ }^{6}$ Indiana University School of Medicine, Indianapolis, IN, USA ${ }^{7}$ VA San Diego Healthcare System, San Diego, CA, USA; ${ }^{8}$ Office of Specialty Care Services, Veterans Health Administration, Washington, DC, USA; ${ }^{9}$ Office of Research \& Development, US Department of Veterans Affairs, Washington, DC, USA; ${ }^{10}$ Division of Medical Genetics, Department of Medicine, VA Greater Los Angeles Healthcare System, Los Angeles, CA, USA; ${ }^{11}$ Department of Medicine, David Geffen School of Medicine at University of California-Los Angeles, Los Angeles, CA, USA; ${ }^{12}$ University of California San Diego, San Diego, CA, USA. Correspondence: Jason L. Vassy (jvassy@partners.org)

\section{REFERENCES}

1. Gammal RS, Caudle KE, Klein TE, Relling MV. Considerations for pharmacogenomic testing in a health system. Genet Med. 2019; https://doi.org/10.1038/s41436-018-0421-x [Epub ahead of print].

2. Caudle KE, Klein TE, Hoffman JM, et al. Incorporation of pharmacogenomics into routine clinical practice: the Clinical Pharmacogenetics Implementation Consortium (CPIC) guideline development process. Curr Drug Metab. 2014;15:209-217.

3. Vassy JL, Stone A, Callaghan JT, et al. Pharmacogenetic testing in the Veterans Health Administration: Policy recommendations from the VHA
Clinical Pharmacogenetics Subcommittee. Genet Med. 2018 Jun 1; https://doi.org/10.1038/s41436-018-0057-x [Epub ahead of print].

4. Martin MA, Hoffman JM, Freimuth RR, et al. Clinical Pharmacogenetics Implementation Consortium guidelines for HLA-B genotype and abacavir dosing: 2014 update. Clin Pharmacol Ther. 2014;95:499-500.

5. Vassy JL, Chun S, Advani S, Ludin SA, Smith JG, Alligood EC. Impact of SLCO1B1 pharmacogenetic testing on patient and healthcare outcomes: a systematic review. Clin Pharmacol Ther. 2018 Aug 23; doi:10.1002/ cpt.1223 [Epub ahead of print].

6. Huitfeldt A, Hernan MA, Kalager M, Robins JM. Comparative effectiveness research using observational data: active comparators to emulate target trials with inactive comparators. EGEMS (Wash DC). 2016;4:1234

7. Mathias PC, Tarczy-Hornoch P, Shirts BH. Modeling the costs of clinical decision support for genomic precision medicine. AMIA Jt Summits Transl Sci Proc. 2016;2016:60-64.

Advance online publication 12 January 2019. doi:10.1038/s41436-018-0422-9 